\title{
PREFACE: SPECIAL ISSUE OF FLOW AND MULTIPHYSICAL TRANSPORT IN POROUS MEDIA
}

This special issue of "Flow and Multiphysical Transport in Porous Media in Special Topics \& Reviews in Porous Media-An International Journal" contains mainly invited papers, whose authors presented their work at one or more of the following three conferences: "The National Conference of Porous Flow of China" and "The International Symposium of Transport in Porous Media," both in Hangzhou on August 24-25, 2017, and "The Chinese Congress of Theoretical and Applied Mechanics" in Beijing on August 15-18, 2017. From the more than 2000 papers submitted, including some 30 keynote lectures, approximately 25 of the highest-quality papers were invited. These papers went through a complete review process. After peer review, seven papers were accepted for publication in this issue.

Contained herein, for theoretical and numerical studies, the article "A Mathematical Model of Microorganisms Adsorption and Desorption in Porous Media" by Xiu et al. presents a three-dimensional, three-phase, nine-component mathematical model to reflect the process of microbial flooding. The model describes the adsorption and reaction kinetics of each component in porous media and the reversible and irreversible adsorption process of bacteria. In addition, the reaction kinetics equation takes into account the effects of nutrients and oxygen. The microorganisms' effect on fluid properties in the reservoir is analyzed in the model, which also elaborates on the key parameters that have important roles in enhancing oil recovery.

In the article "Fluid Flow Path Preferential Selection Algorithm Based on Topological Network Model of Porous Media," Zhang et al. present an equivalent topological network model to describe fluid-seepage properties and path selection by complex circuits in a series-parallel connection. The authors apply this model to a quantitative analysis of the geometric structure's influence on fluid flow and flow path selection. The paper describes the equivalent network model and optimization algorithm for analyzing flow process and flow quantity distribution. After validation by numerical simulations, the authors show that under the circumstances only the geometric structure of porous media is known, the equivalent topological network model and flow path preferential selection algorithm can describe the fluid flow in porous media, and flow path selection behavior can be predicted.

The article "Research on Dynamic Change of Reservoir Permeability Based on Coupled Pore Fluid Flow and Stress Analysis" by Han et al. studies the coupling of pore fluid flow and stress analysis of reservoirs. On the basis of rock mechanics and seepage mechanics theory, governing equations of coupled pore fluid flow and stress are given for the injection and production of a tight sandstone reservoir. The mathematical model established for injection and production of the tight sandstone reservoir describes the dynamic changes in permeability in the form of variations in reservoir temperature, pore pressure, and bulk strain. Numerical simulation results of coupled pore fluid flow and stress in the injection and production of the reservoir mimic the actual production situation, which impacts significantly on oil-field development.

In the article "Electrokinetic Coupling Model for Porous Flow in Tight Oil Reservoirs," Xiao et al. develop a new electrokinetic coupling model based on correcting the viscosity of electrical double layer fluid, the theory of HagenPoiseuille flow, and electro-osmotic flow. The calculations show that the volumetric flow rate of the electrokinetic coupling model is considerably smaller than that of the Hagen-Poiseuille flow when no applied direct current (DC) electric field is present. Adverse electro-osmosis is one of the important factors that weaken the volumetric flow rate, which is significantly reduced with increased surface-to-volume ratio. The rate can be increased considerably more effectively by an appropriately applied DC electric field combined with pressure.

The article "A Heat and Mass Transfer Coupling Model for Transition Interface of Expanding Solvent SteamAssisted Gravity Drainage" by Chang et al. develops a heat and mass transfer coupling model for the transition interface of multicomponent expanding solvent steam-assisted gravity drainage. This model integrates both the equation of state and the fugacity equation to calculate the transition-interface equilibrium state. Results show that increasing the 
fraction of the lighter solvent can accelerate mass transfer, but the heat that is transferred is seriously deteriorated. In addition, the closer the stream's bubble point temperature and dew point temperature, the smaller the elevation of mass transfer, whereas heat transfer is almost unaffected. Therefore, to achieve optimum effects of heat and mass transfer, solvents with a larger saturation temperature difference must be chosen, and the composition must be optimized based on the formation of thermal properties and solvent components.

In the article "Study on Water-Flooding Performance in Low-Permeability Reservoirs using the Stream Tube Method," Yin et al. develop a mathematical model of a five-spot pattern using stream tubes. For verification, the oil rate that is calculated using this method is shown to be consistent with the actual data. An analysis of the dynamic changes of calculated oil rate, water rate, oil recovery, and sweep efficiency, combined with the displacement front, shows that water breakthrough in the stream tube results in changes in performance results. The authors show that the smaller the mobility ratio, the slower the injected water spreads, the longer the water breakthrough time of the producer, and the bigger the oil recovery during a period of time after breakthrough. With decreasing injector-producer distance, the spread of the injected water augments, so water breakthrough time decreases, and oil recovery increases after a certain breakthrough time.

Finally, in the article "Multiple Scenarios Integrated Upscaling with Full-Tensor Effects of Fractured Reservoirs," Lei et al. develops a new transmissibility upscaling approach to effectively capture full-tensor effects when upscaling from discrete fractured models based on a global upscaling technique with an output least-squares method. The authors propose to compute a coarse scale transmissibility field using multipoint flux approximations that allow for full-tensor effects that can be generally applied to variable flow situations. The new approach yields a robust performance when used in different simulation scenarios.

The Guest Editors thank the authors of the papers for their scientific contributions and the article reviewers for their time and effort to ensure that the high standards for submitted manuscripts are maintained. We are also grateful to Begell House (the Home of Sciences and Engineering) and its team for their work in the publication of this special issue. Finally, we are grateful to the Editor-in-Chief of the journal, Kambiz Vafai, for his kind contributions throughout the entire process.

\section{Guest Editors:}

\author{
Moran Wang \\ Tsinghua University \\ Beijing, China
}

Boming Yu

Huazhong University of Science and Technology

Wuhan, China
Jun Yao

China Petroleum University

Qingdao, China

Kambiz Vafai

University of California

Riverside, California, USA 